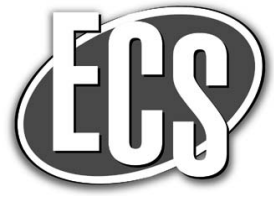

\title{
Efficient Simulation and Model Reformulation of Two-Dimensional Electrochemical Thermal Behavior of Lithium-Ion Batteries
}

\author{
Paul W. C. Northrop, , ,* Manan Pathak,,** Derek Rife, ${ }^{\mathrm{c}, *}$ Sumitava De, \\ Shriram Santhanagopalan, ${ }^{\mathrm{d}, * *}$ and Venkat R. Subramanian ${ }^{\mathrm{b}, \mathrm{e}, * *, z}$ \\ ${ }^{a}$ CFD Research Corporation, Biomedical and Energy Technologies, Huntsville, Alabama 35806, USA \\ ${ }^{b}$ University of Washington, Seattle, Washington 98105, USA \\ ${ }^{c}$ Department of Energy, Environmental and Chemical Engineering, Washington University, Saint Louis, \\ Missouri 63130, USA \\ ${ }^{d}$ Transportation and Hydrogen Systems Center, National Renewable Energy Laboratory, Golden, Colorado 80401, USA \\ ${ }^{e}$ Pacific Northwest National Laboratory, Richland, Washington 99352, USA
}

\begin{abstract}
Lithium-ion batteries are an important technology to facilitate efficient energy storage and enable a shift from petroleum based energy to more environmentally benign sources. Such systems can be utilized most efficiently if good understanding of performance can be achieved for a range of operating conditions. Mathematical models can be useful to predict battery behavior to allow for optimization of design and control. An analytical solution is ideally preferred to solve the equations of a mathematical model, as it eliminates the error that arises when using numerical techniques and is usually computationally cheap. An analytical solution provides insight into the behavior of the system and also explicitly shows the effects of different parameters on the behavior. However, most engineering models, including the majority of battery models, cannot be solved analytically due to non-linearities in the equations and state dependent transport and kinetic parameters. The numerical method used to solve the system of equations describing a battery operation can have a significant impact on the computational cost of the simulation. In this paper, a model reformulation of the porous electrode pseudo three dimensional (P3D) which significantly reduces the computational cost of lithium ion battery simulation, while maintaining high accuracy, is discussed. This reformulation enables the use of the P3D model into applications that would otherwise be too computationally expensive to justify its use, such as online control, optimization, and parameter estimation. Furthermore, the P3D model has proven to be robust enough to allow for the inclusion of additional physical phenomena as understanding improves. In this paper, the reformulated model is used to allow for more complicated physical phenomena to be considered for study, including thermal effects. (c) The Author(s) 2015. Published by ECS. This is an open access article distributed under the terms of the Creative Commons Attribution Non-Commercial No Derivatives 4.0 License (CC BY-NC-ND, http://creativecommons.org/licenses/by-nc-nd/4.0/), which permits non-commercial reuse, distribution, and reproduction in any medium, provided the original work is not changed in any way and is properly cited. For permission for commercial reuse, please email: oa @electrochem.org. [DOI: 10.1149/2.0341506jes] All rights reserved.
\end{abstract}

Manuscript submitted August 19, 2014; revised manuscript received January 19, 2015. Published March 9, 2015.

There is an increasing societal pressure to utilize alternative energy sources to supplant the high use of fossil fuels. As energy and power demand is continually increasing, both in terms of grid usage and for transportation, there has been more interest in developing renewable energy sources. One problem with renewable energy sources is the intermittent nature and short-term unpredictability of supply of sources such as wind and solar. Thus, in order to match supply and demand, some form of energy storage is required, and lithium-ion battery technologies are one possible solution. Furthermore, electric vehicles are increasing in popularity as the price of liquid fuels generally increase. Lithium-ion batteries are a popular choice for electric vehicles because of their high energy and power density compared to other battery chemistries.

The performance of lithium-ion batteries is highly dependent on the conditions at which they are exposed as well as the state of the internal variables. This has led to the development of several mathematical models to simulate battery behavior, ranging from simple empirical-based models or circuit based models ${ }^{1,2}$ to computationally expensive molecular dynamics simulators (which cannot be practically used to simulate a full cell). Much of the research on battery simulation has focused on continuum-scale models that exist between the two extremes, most notably the single particle model (SPM) and the pseudo two-dimensional (P2D) porous electrode model..$^{3-7}$ The SPM neglects variation across the thickness of the electrode and ignores the effects of the electrolyte phase, which enables fast computation. ${ }^{8-11}$ In contrast, the P2D model tracks the lithium concentration in the active material and electrolyte as well as the potential in the electrolyte and the active material. ${ }^{3,12}$ Furthermore, the P2D model developed by Doyle et al. ${ }^{3}$ has proven to be robust enough to allow for the inclusion of additional physical phenomena as understanding improves, ${ }^{6,10,12-21}$ for example, by considering solid electrolyte in-

\footnotetext{
*Electrochemical Society Student Member

**Electrochemical Society Active Member.

zE-mail: vsubram@uw.edu
}

terface (SEI) layer growth ${ }^{8,19,22}$ or the stress development in active particles during lithium intercalation. ${ }^{23-29}$

Performance, safety, and life behavior are directly impacted by the temperature which a cell experiences during cycling. ${ }^{30}$ For instance, low temperatures compromise the amount of power that a battery can provide by increasing the resistance to lithium diffusion in the cell. This makes operating battery powered devices difficult in cold weather. Conversely, a battery operated at a high temperature can be physically damaged or experience higher levels of capacity fade. ${ }^{30}$ An overheated or overcharged cell may ultimately undergo thermal runaway, a potentially explosive situation. Although thermal runaway is a severe, acute event, high operating temperatures can also have other deleterious effects. ${ }^{30}$ Side reactions which contribute to capacity fade may be more favorable under high temperatures. ${ }^{31}$ Stresses caused by thermal expansion can contribute to mechanical failures. Vaporization of the electrolyte can cause pressure buildup which can lead to rupture and expose the (highly reactive) battery internals to the atmosphere. Predicting and maintaining temperature rises in a lithium ion cell is critical to ensure safety and life, especially in high power applications which can see rather large temperature rises. Thus, considering the thermal effects in lithium-ion batteries has been a popular subject in the literature when designing and operating batteries. ${ }^{32-36}$

The P2D model is popular among researchers due to its good predictive capability, but also has higher computational costs compared to the single particle or circuit based models. The P2D model is often solved using the method of lines (MOL) by discretizing the spatial dimensions ( $x$ and $r$ ) using numerical techniques (often finite difference) to convert the system of PDEs to a system of $1^{\text {st }}$ order differential algebraic equations (DAEs). ${ }^{37-40}$ The MOL allows for computationally efficient time stepping algorithms, such as DASSL and DASKR, ${ }^{41}$ to be used to simulate the model. This is an initial value problem, which requires initial conditions to be provided that must be consistent with the algebraic equations, leading many DAE solvers to fail when applied to battery models if consistent initial conditions are not provided. ${ }^{5,42}$ 
Table I. Governing equations of the porous electrode model in higher dimensions.

Governing Equations

$$
\begin{aligned}
& \varepsilon_{i} \frac{\partial c}{\partial t}=\nabla \cdot D_{e f f, i} \nabla c+a_{\mathrm{p}}\left(1-t_{+}\right) j_{i} \\
& -\nabla \cdot\left[\sigma_{e f f} \nabla \Phi_{1}\right]-\nabla \cdot\left[\kappa_{\mathrm{eff}} \nabla \Phi_{2}\right]+\nabla \cdot\left[\frac{2 \kappa_{\mathrm{eff}} R T}{F}\left(1-t_{+}\right) \nabla \ln c\right]=0 \\
& \sigma_{e f f, i} \nabla^{2} \Phi_{1}=a_{i} F j_{i} \\
& \frac{\partial c_{p}^{s}}{\partial t}=\frac{1}{r^{2}} \frac{\partial}{\partial r}\left[r^{2} D_{p}^{s} \frac{\partial c_{p}^{s}}{\partial r}\right] \\
& \rho_{i} C_{p, \mathrm{i}} \frac{d T_{p}}{d t}=\nabla \cdot \lambda_{i} \nabla T+Q
\end{aligned}
$$

The high computational cost of P2D simulation has motivated researchers to develop mathematical techniques to improve simulation efficiency. Several researchers have used different discretization schemes to eliminate the radial dependence and reduce the dimensionality by one..$^{3,39,43-46}$ Attempts to simplify the primary dimension have also been done to reduce the computational cost. For example, proper orthogonal decomposition (POD) can reduce the total number of states simulated, but the system must be recreated if parameters or operating conditions are changed. ${ }^{47}$ Quasi-linearization combined with a Padé approximation has also been used to simplify the model. ${ }^{48}$ Reformulation work based on Galerkin's method has been used but was unable to handle non-linear parameters. ${ }^{38}$ However, a model reformulation based on orthogonal collocation has proven to be accurate, fast, and robust for simulating battery performance. ${ }^{40}$ Reformulation based on orthogonal collocation has enabled the P2D model to be used for parameter estimation ${ }^{49}$ optimization,${ }^{50}$ and real-time control. ${ }^{51}$

The standard porous electrode P2D model is a one dimensional model (the pseudo dimension refers to the radial direction in the solid phase particles). However, in large format cells, such as those which are used in electric vehicles, grid storage, or in satellites, a 1D model may not be adequate as the variation in the direction parallel can become significant. This is especially important when temperature effects are considered. For example, a large cell can experience a large temperature rise in the center of the electrode relative to the surface, which directly affects the local current density, diffusion rates, and other phenomena. For clarity and to convey the variation in the $y$ direction, in this paper, we will call this model a Pseudo-3D (P3D) model. The porous electrode model generalized to higher dimensions is given in the Table I.

The highly detailed nature of the standard P2D model makes the computation rather difficult, while simulating a P3D model greatly increases the computational expense. A finite difference approach with 50 node points in each electrode and 35 node points in the separator in the $x$-direction and 25 node points in the $y$-direction results in a system of nearly 15,000 DAEs that must be solved for a single cell, if the parabolic profile is used in the solid phase. If a more detailed discretization is used for the solid-phase diffusion, the number of equations will be even greater. Thus, much of the work done by researchers modeling thermal behavior in batteries have used a 1D model ${ }^{30}$ or decoupled the thermal profile from the electrochemical reactions. ${ }^{4,35,36,48,52,53}$ For example, Evans et al. ${ }^{52}$ modeled heat generation in cylindrical cells using local current density which was decoupled from the overall thermal effects. Kim et al. ${ }^{54}$ simulated a large format lithium-ion polymer battery using parameters from small cells, but maintained a one-way coupling between the thermal and electrochemical effects (i.e. the electrochemical reactions and current flow contributed to the thermal calculation, but the temperature did not affect electrochemical behavior). They extended this model for electric vehicle applications using constant power charge/discharge protocols. ${ }^{55}$ Inui et al. ${ }^{56}$ studied heat generation in prismatic and cylindrical cells using a finite element analysis with resistive heating in each element. Kim et al. ${ }^{57}$ developed a Multi-Scale MultiDimensional (MSMD) model to study large format lithium-ion bat- teries. This MSMD model simulated the electrochemical behavior at the particle, electrode, and cell domains, with appropriate coupling between the scales. This allowed multiple cell designs to be tested, while only using a 1D model for the electrode domain (analogous to cell sandwich level), but being able to model the temperature in $3 \mathrm{D}^{57}$

Gerver and Meyers ${ }^{58}$ performed 3D thermal simulation of lithium batteries in planar configurations by arranging several 1D porous electrode models in series and a grid configuration. Thus, all current flow in the cell sandwich was in the direction perpendicular to the electrode, and lithium-ion transport in the direction parallel to the electrodes was neglected. The different $1 \mathrm{D}$ nodes were coupled at the current collectors, which were modeled as a system of temperature varying resistors. ${ }^{58}$ This improves the computational efficiency as solving several 1D models is simpler than solving a full 2- or 3-dimensional model, while allowing for some effects of a temperature profile to be analyzed. McCleary et al. ${ }^{59}$ extended the work of Gerver and Meyers by applying a similar approach of using a series of $1 \mathrm{D}$ porous electrode models to spirally and prismatically wound cells, which are standard configurations for commercially made lithium-ion cells, by accounting for the effects of curvature and increasing electrode surface areas in the outer layers. They were able to model the effect of the number and positions of tabs on heat generation but again neglected ion transport and current density in the direction parallel to the electrodes. ${ }^{59}$ Christensen et al. ${ }^{60}$ coupled the $1 \mathrm{D}$ dualfoil model ${ }^{34}$ in a Fluent environment, ${ }^{61}$ allowing a fine mesh grid to be used for the temperature simulation while using a coarser mesh for the electrochemical reactions. At each time step, the local temperature was used to determine the behavior of each electrochemical element by using a Newton-Raphson approach to solve for the voltage to achieve the total specified current (with voltage and temperature being the only variable to couple the 1D electrochemical elements). Once the electrochemical elements reached a converged solution, the heat generation was calculated from the current density and fed into the thermal mesh. ${ }^{60}$ This approach allowed parallelization of a multi-core processor performed to solve the system improving the computational speed of the entire system. ${ }^{60}$ Tourani et al. ${ }^{62}$ coupled a series of $1 D$ porous electrode models while simulating a 2D thermal model, with heat generation occurring due to the electrochemical reaction and electrical resistances and experimentally verified their results for a lithium polymer cell, and a lithium iron phosphate cell. Xu et al ${ }^{63}$ performed a two-dimensional modeling using COMSOL ${ }^{64}$ which accounted for mass and charge conservation in both dimensions and examined the effect that tab position had on the temperature profiles.

\section{Reformulation and Simulation}

In this work, a fully coupled thermal-electrochemical battery model is developed which accounts for a two dimensional variation of all dependent variables. As noted previously, such a model can be prohibitively expensive to directly simulate. Wu et al. ${ }^{67}$ showed the importance of understanding the physics of the problem and the effect of the extent of coupling of the potential equations on simulation times using a similar battery model. They developed an algorithm based on the Newton linearization scheme and solved the resulting algebraic equations using an iterative GMRES solver rather than the usual Gauss elimination and were able to show an increase in the convergence speed. In addition, they showed that Jacobian estimation need not be exact, but representative of the problem with the most significant physics involved captured in the Jacobian estimation. Note that their paper uses finite volume method which conserves mass, momentum and in our opinion, this helps avoid the need for accurate Jacobian estimation. Jacobian estimation accuracy can depend on the solver and method used in time and also the method used for discretization.

However, in order to reduce the computational cost even further, here we use orthogonal collocation in two dimensions in a method analogous to that used in model reformulations in 1D as reported earlier. ${ }^{40}$ This allows the simulation of a porous electrode pseudo 3D (P3D) model with thermal effects to be performed in a reasonable 
time. Table I shows how including additional dimensions make simulation more complicated. Specifically, the charge balance must be represented as a $2^{\text {nd }}$ order PDE in the $2 \mathrm{D}$ formulation. When only a single dimension is considered, the charge balance equation can be simply integrated to give the total current flowing through the cell, resulting in a $1^{\text {st }}$ order PDE often used in the one-dimensional formulation of the porous electrode model.

As mentioned previously in Northrop et al., ${ }^{40}$ the original model equations are defined in three regions sequentially, namely cathode, separator and anode. For example, the positive electrode equations are defined in the region $\left[0, l_{p}\right]$ whereas the negative electrode equations are defined in the region $\left[l_{p}+l_{s}, l_{p}+l_{s}+l_{n}\right]$. In order to keep the computations simple, each of the coordinates in both $x$ and $y$ directions are transformed to the interval $[0,1]$. A list of transformed equations is shown in Table II for each of the three regions.

Orthogonal collocation is used here to discretize the spatial direction to develop a system of differential algebraic equations (DAEs), which can be solved using efficient and well-established integrators with variable time steps. The application of orthogonal collocation to higher dimensions is a logical extension of the one-dimensional case, which can be found in Ref. 40, and is described in detail in Villadsen and Michelsen. ${ }^{65}$ However, rather than estimating each dependent variable as a series solution with a single summation, the series approximation must consist of a double summation of trial functions which can generally be given as

$$
u_{\text {approx }}(x, y, t)=\sum_{j=0}^{N+2} \sum_{k=0}^{N+2} B_{j, k}(t) Z_{j}(y) Z_{k}(x)
$$

Where $Z_{j}(y)$ and $Z_{k}(x)$ are pre-chosen trial functions. The first $N+1$ coefficients, $B_{j, k}(t)(j, k=0$ to $N)$, are determined by using the method of weighted residuals (MWR) which aims to find the coefficients which minimize the error. ${ }^{52}$ The coefficients corresponding to $j, k=N+1$ and $N+2$ are calculated using the boundary conditions and will be discussed shortly. First, consider a general differential equation of the form (for example, the governing equations given in Table II):

$$
D[u(x, y, t)]=0
$$

Define the residual of an approximate solution as

$$
R\left(x, y, B_{j, k}(t)\right)=D\left[u_{\text {approx }}(x, y, t)\right]
$$

which describes how much the approximate solution does not exactly satisfy the system of differential equations. If the residual, $R(x, y)$, is identically zero for all $x$ and $y$, the approximate solution is the exact solution, but this is not generally the case in numerical solutions. The MWR aims to minimize the residual across the domain in some average way:

$$
\iint R\left(x, y, B_{j, k}(t)\right) W_{j, k}(x, y) d x d y=0
$$

Where enough weight functions, $W_{j, k}(x)$, are used to generate enough equations to solve for the unknown coefficients, $B_{j, k}(t)$. In the case of highly non-linear governing equations, as those found in battery models, the integration in Equation 4 must be done numerically with significant computational cost. For collocation, the Dirac delta function is used as the weight function, $W_{j, k}(x)=\delta\left(x-x_{j}\right) \delta\left(y-y_{k}\right)$, to eliminate the need to perform the integration and the system reduces to

$$
R\left(B_{j, k}(t), x=x_{j}, y=y_{k}\right)=0
$$

Equation 5 is a system of DAEs in which the $x$ and $y$ dependence has been eliminated, and can be used to solve for the $B_{j, k}(t)$ coefficients.

Importantly, the error is minimized when the collocation points are chosen specifically as zeros of orthogonal polynomials. This is referred to as orthogonal collocation. ${ }^{53}$ In this work, the trial functions used are Chebyshev polynomials shifted to the domain $[0,1]$ (rather than $[-1,1]$ as Chebyshev polynomials are traditionally defined). Thus, the trial functions are given as:

$$
Z_{j}(X)=T_{j}(2 X-1)
$$

The collocation points used are the Chebyshev-Gauss-Lobatto (CGL) points, of which the $k^{\text {th }}$ zero is given by

$$
X_{k, N}=-\frac{1}{2} \cos \left(\frac{(k+1 / 2) \pi}{N+1}\right)+\frac{1}{2}
$$

When $N$ trial functions are used. Note that equations 6 and 7 apply to the $y$-direction as well.

Determining the coefficients, $B_{j, k}(t)$ corresponding to $j, k=N+1$ and $N+2$ can be done in a similar manner as described above. However, as the boundaries are one dimensional, it is somewhat simpler. Consider the general boundary condition at $x=0$ (with the understanding that the same procedure applies to the other boundary):

$$
D_{B C}[u(x=0, y, t)]=0
$$

Again, the residual is used

$$
R_{B C}\left(y, B_{j, k}(t)\right)=D_{B C}\left[u_{\text {approx }}(x=0, y, t)\right]
$$

Rather than the double integral used in Equation 4, only a single integral is required as the $x$ dependence is eliminated at the boundary.

$$
\int R_{B C}\left(y, B_{j, k}(t)\right) W_{k}(y) d y=0
$$

Again, Dirac delta functions are used to eliminate the need to compute the integration, $W_{k}(x)=\delta\left(y-y_{k}\right)$. Thus the discretized system of equations arising from the boundary conditions becomes

$$
R_{B C}\left(B_{j, k}(t), y=y_{k}\right)=0
$$

Unlike Equation 5, Equation 11 is a system of algebraic equations with the spatial dependence eliminated. Furthermore, if the boundary conditions are linear (as they are in the battery model), Equation 11 can be used to solve some of the $B_{j, k}(t)$ coefficients prior to implementing the main DAE solver. This reduces the overall computation cost of the simulation.

By applying the approximation given in Equation 1 to the governing equations in Table I, a system of DAEs is developed with time as the only independent variable. As the series solution is infinitely differentiable, all the derivative and gradient terms can be analytically determined and eliminated. This system can be solved using efficient time-adaptive solvers, and a converged solution can be achieved while using fewer terms than a finite difference discretization.

Two-dimensional stack with simplified boundary conditions.One challenge of the two dimensional modeling of lithium ion batteries is the determination and application of appropriate boundary conditions of the solid phase potential, $\Phi_{1}$, at the current collector/ electrode interface. Note that the current flowing out of the cell is calculated from the derivative of $\Phi_{1}$, in a manner analogous to heat transfer. In a one dimensional model, the flux of $\Phi_{1}$ can thus be specified at the boundary. However, in a multidimensional model, such an approach neglects the possible variation parallel to the electrodes. For example, more current may flow out of the top of the cell than out of the bottom. In this section we assume that the current density across the height of the cell is constant as a first approximation.

In this subsection, we consider the boundary conditions as given in Figure 1, with the realization that the solid phase boundary conditions neglects the possible variation of current in the $y$-direction, but is used as initial approximation of a 2D model. Relaxations of this assumption are discussed later. Note that the continuity boundary conditions are applied at the electrode/separator interfaces. For all simulations performed in this work, the supplemental equations given in Table III are used to calculate heat generation and kinetics, as well as thermal dependence. Similarly, Table IV gives the parameter values used in these models.

Note also that the boundary conditions for temperature are not identical at the top and bottom of the cell, with the bottom being at a specified cold plate temperature while the top is insulated. This breaks the symmetry in the $y$-direction and forces variations to exist in $y$. The 


\section{Table II. Transformed Governing Equations for Li-ion batteries.}

Governing Equation

Positive Electrode

$\varepsilon_{\mathrm{p}} \frac{\partial c_{p}}{\partial t}=\frac{1}{l_{p}} \frac{\partial}{\partial X}\left[\frac{D_{\mathrm{eff}, \mathrm{p}}}{l_{p}} \frac{\partial c_{p}}{\partial X}\right]+\frac{1}{l_{y}} \frac{\partial}{\partial Y}\left[\frac{D_{\mathrm{eff}, \mathrm{p}}}{l_{y}} \frac{\partial c_{p}}{\partial Y}\right]+a_{\mathrm{p}}\left(1-t_{+}\right) j_{\mathrm{p}}$

$\frac{-\sigma_{\text {eff,p }}}{l_{p}{ }^{2}} \frac{\partial\left(\frac{\partial \Phi_{1, p}}{\partial X}\right)}{\partial X}-\frac{\sigma_{\text {eff,p }}}{l_{y}{ }^{2}} \frac{\partial\left(\frac{\partial \Phi_{1, p}}{\partial Y}\right)}{\partial Y}-\frac{\kappa_{\text {eff,p }}}{l_{p}{ }^{2}} \frac{\partial\left(\frac{\partial \Phi_{2, p}}{\partial X}\right)}{\partial X}-\frac{\kappa_{\text {eff,p }}}{l_{y}{ }^{2}} \frac{\partial\left(\frac{\partial \Phi_{2, p}}{\partial Y}\right)}{\partial Y}$

$+\frac{2 \kappa_{\mathrm{eff}, \mathrm{p}} R T}{F} \frac{\left(1-t_{+}\right)}{l_{p}{ }^{2}} \frac{\partial\left(\frac{\partial \ln c_{p}}{\partial X}\right)}{\partial X}+\frac{2 \kappa_{\mathrm{eff}, \mathrm{p}} R T}{F} \frac{\left(1-t_{+}\right)}{l_{y}{ }^{2}} \frac{\partial\left(\frac{\partial \ln c_{p}}{\partial Y}\right)}{\partial Y}=0$

$\frac{1}{l_{p}} \frac{\partial}{\partial X}\left[\frac{\sigma_{\mathrm{eff}, \mathrm{p}}}{l_{p}} \frac{\partial}{\partial X} \Phi_{1, p}\right]+\frac{1}{l_{y}} \frac{\partial}{\partial Y}\left[\frac{\sigma_{\mathrm{eff}, \mathrm{p}}}{l_{y}} \frac{\partial}{\partial Y} \Phi_{1, p}\right]=a_{\mathrm{p}} F j_{\mathrm{p}}$

$\frac{\partial c_{p}^{s}}{\partial t}=\frac{1}{r^{2}} \frac{\partial}{\partial r}\left[r^{2} D_{p}^{s} \frac{\partial c_{p}^{s}}{\partial r}\right]$

$\rho_{p} C_{p, p} \frac{d T_{p}}{d t}=\frac{1}{l_{p}} \frac{\partial}{\partial X}\left[\frac{\lambda_{p}}{l_{p}} \frac{\partial T_{p}}{\partial X}\right]+\frac{1}{l_{y}} \frac{\partial}{\partial Y}\left[\frac{\lambda_{p}}{l_{y}} \frac{\partial T_{p}}{\partial Y}\right]$

$+Q_{\mathrm{rxn}, \mathrm{p}}+Q_{\mathrm{rev}, \mathrm{p}}+Q_{\mathrm{ohm}, \mathrm{p}}$

Separator

$\varepsilon_{s} \frac{\partial c_{s}}{\partial t}=\frac{1}{l_{s}} \frac{\partial}{\partial X}\left[\frac{D_{\mathrm{eff}, \mathrm{s}}}{l_{s}} \frac{\partial c_{s}}{\partial X}\right]+\frac{1}{l_{y}} \frac{\partial}{\partial Y}\left[\frac{D_{\mathrm{eff}, \mathrm{s}}}{l_{y}} \frac{\partial c_{s}}{\partial Y}\right]$

$-\frac{\kappa_{\mathrm{eff}, \mathrm{s}}}{l_{s}^{2}} \frac{\partial\left(\frac{\partial \Phi_{2, s}}{\partial X}\right)}{\partial X}-\frac{\kappa_{\mathrm{eff}, \mathrm{s}}}{l_{y}{ }^{2}} \frac{\partial\left(\frac{\partial \Phi_{2, s}}{\partial Y}\right)}{\partial Y}+\frac{2 \kappa_{\mathrm{eff}, \mathrm{s}} R T}{F} \frac{\left(1-t_{+}\right)}{l_{s}{ }^{2}} \frac{\partial\left(\frac{\partial \ln c_{S}}{\partial X}\right)}{\partial X}$

$+\frac{2 \kappa_{\mathrm{eff}, \mathrm{s}} R T}{F} \frac{\left(1-t_{+}\right)}{l_{y}^{2}} \frac{\partial\left(\frac{\partial \ln c_{s}}{\partial Y}\right)}{\partial Y}=0$

$\rho_{s} C_{p, s} \frac{d T_{s}}{d t}=\frac{1}{l_{s}} \frac{\partial}{\partial X}\left[\frac{\lambda_{s}}{l_{s}} \frac{\partial T_{s}}{\partial X}\right]+\frac{1}{l_{s}} \frac{\partial}{\partial Y}\left[\frac{\lambda_{s}}{l_{y}} \frac{\partial T_{s}}{\partial Y}\right]+Q_{\mathrm{ohm}, \mathrm{s}}$

Boundary Conditions

$$
\begin{aligned}
& \left.\frac{\partial c_{p}}{\partial X}\right|_{X=0}=0 \\
& \left.\frac{-D_{\text {eff,p }}}{l_{p}} \frac{\partial c_{p}}{\partial X}\right|_{X=1}=\left.\frac{-D_{\text {eff, }}}{l_{s}} \frac{\partial c_{s}}{\partial X}\right|_{X=0} \\
& \left.\frac{\partial c_{p}}{\partial Y}\right|_{Y=0}=0 \\
& \left.\frac{\partial c_{p}}{\partial Y}\right|_{Y=1}=0 \\
& \left.\frac{\partial \Phi_{2, p}}{\partial X}\right|_{X=0}=0 \\
& \left.\frac{-\kappa_{\mathrm{eff}, \mathrm{p}}}{l_{p}} \frac{\partial \Phi_{2, p}}{\partial X}\right|_{X=1}=\left.\frac{-\kappa_{\mathrm{eff}, \mathrm{s}}}{l_{s}} \frac{\partial \Phi_{2, s}}{\partial X}\right|_{X=0} \\
& \left.\frac{\partial \Phi_{2, p}}{\partial Y}\right|_{Y=0}=0 \\
& \left.\frac{\partial \Phi_{2, p}}{\partial Y}\right|_{Y=1}=0 \\
& \left.l_{y} \int_{0}^{1}\left(\frac{1}{l_{p}} \frac{\partial \Phi_{1, p}}{\partial X}\right) d Y\right|_{X=0}=-\frac{I}{\sigma_{e f f, p}} \\
& \left.\frac{\partial \Phi_{1, p}}{\partial X}\right|_{X=1}=0 \\
& \left.\frac{\partial \Phi_{1, p}}{\partial Y}\right|_{Y=0}=0 \\
& \left.\frac{\partial \Phi_{1, p}}{\partial Y}\right|_{Y=1}=0 \\
& \left.\frac{\partial c_{p}^{s}}{\partial r}\right|_{r=0}=0 \\
& -\left.D_{p}^{s} \frac{\partial c_{p}^{s}}{\partial r}\right|_{r=R_{s}}=j_{p} \\
& \left.\frac{\partial T_{p}}{\partial X}\right|_{X=0}=0 \\
& -\left.\frac{\lambda_{p}}{l_{p}} \frac{\partial T_{p}}{\partial X}\right|_{X=1}=-\left.\frac{\lambda_{s}}{l_{s}} \frac{\partial T_{s}}{\partial X}\right|_{X=0} \\
& \left.T_{p}\right|_{Y=0}=293 K \\
& \left.\frac{\partial T_{p}}{\partial Y}\right|_{Y=1}=0 \\
& \left.c_{p}\right|_{X=1}=\left.c_{s}\right|_{X=0} \\
& \left.c_{S}\right|_{X=1}=\left.c_{n}\right|_{X=0} \\
& \left.\frac{\partial c_{s}}{\partial Y}\right|_{Y=0}=0 \\
& \left.\frac{\partial c_{s}}{\partial Y}\right|_{Y=1}=0 \\
& \left.\Phi_{2, p}\right|_{X=1}=\left.\Phi_{2, s}\right|_{X=0} \\
& \left.\Phi_{2, s}\right|_{X=1}=\left.\Phi_{2, n}\right|_{X=0} \\
& \left.\frac{\partial \Phi_{2, s}}{\partial Y}\right|_{Y=0}=0 \\
& \left.\frac{\partial \Phi_{2, s}}{\partial Y}\right|_{Y=1}=0 \\
& \left.T_{p}\right|_{X=1}=\left.T_{s}\right|_{X=0} \\
& \left.T_{s}\right|_{X=0}=\left.T_{n}\right|_{X=1} \\
& \left.T_{S}\right|_{Y=0}=293 K \\
& \left.\frac{\partial T_{s}}{\partial Y}\right|_{Y=1}=0
\end{aligned}
$$




\section{Table II. (Continued.)}

Governing Equation

Boundary Conditions

Negative Electrode

$\varepsilon_{n} \frac{\partial c_{n}}{\partial t}=\frac{1}{l_{n}} \frac{\partial}{\partial X}\left[\frac{D_{\text {eff, }}}{l_{n}} \frac{\partial c_{n}}{\partial X}\right]+\frac{1}{l_{y}} \frac{\partial}{\partial Y}\left[\frac{D_{\text {eff, }}}{l_{y}} \frac{\partial c_{n}}{\partial Y}\right]+a_{n}\left(1-t_{+}\right) j_{n}$

$\left.\frac{\partial c_{n}}{\partial X}\right|_{X=1}=0$

$\left.\frac{-D_{\text {eff,s }}}{l_{s}} \frac{\partial c_{s}}{\partial X}\right|_{X=1}=\left.\frac{-D_{\text {eff, } \mathrm{n}}}{l_{n}} \frac{\partial c_{n}}{\partial X}\right|_{X=0}$

$\left.\frac{\partial c_{n}}{\partial Y}\right|_{Y=0}=0$

$\left.\frac{\partial c_{n}}{\partial Y}\right|_{Y=1}=0$

$\frac{-\sigma_{\text {eff, } \mathrm{n}}}{l_{n}{ }^{2}} \frac{\partial\left(\frac{\partial \Phi_{1, n}}{\partial X}\right)}{\partial X}-\frac{\sigma_{\text {eff, }}}{l_{y}{ }^{2}} \frac{\partial\left(\frac{\partial \Phi_{1, n}}{\partial Y}\right)}{\partial Y}-\frac{\kappa_{\text {eff,n }}}{l_{n}{ }^{2}} \frac{\partial\left(\frac{\partial \Phi_{2, n}}{\partial X}\right)}{\partial X}-\frac{\kappa_{\text {eff, }}}{l_{y}{ }^{2}} \frac{\partial\left(\frac{\partial \Phi_{2, n}}{\partial Y}\right)}{\partial Y}$

$\left.\Phi_{2, n}\right|_{X=1}=0$

$+\frac{2 \kappa_{\mathrm{eff}, \mathrm{n}} R T}{F} \frac{\left(1-t_{+}\right)}{l_{n}{ }^{2}} \frac{\partial\left(\frac{\partial \ln c_{n}}{\partial X}\right)}{\partial X}+\frac{2 \kappa_{\mathrm{eff}, \mathrm{n}} R T}{F} \frac{\left(1-t_{+}\right)}{l_{y}{ }^{2}} \frac{\partial\left(\frac{\partial \ln c_{n}}{\partial Y}\right)}{\partial Y}=0$

$\left.\frac{-\kappa_{\text {eff, } \mathrm{s}}}{l_{s}} \frac{\partial \Phi_{2, s}}{\partial X}\right|_{X=1}=\left.\frac{-\kappa_{\text {eff, }}}{l_{n}} \frac{\partial \Phi_{2, n}}{\partial X}\right|_{X=0}$

$\left.\frac{\partial \Phi_{2, n}}{\partial Y}\right|_{Y=0}=0$

$\left.\frac{\partial \Phi_{2, n}}{\partial Y}\right|_{Y=1}=0$

$\frac{1}{l_{n}} \frac{\partial}{\partial X}\left[\frac{\sigma_{\mathrm{eff}, \mathrm{n}}}{l_{n}} \frac{\partial}{\partial X} \Phi_{1, n}\right]+\frac{1}{l_{y}} \frac{\partial}{\partial Y}\left[\frac{\sigma_{\mathrm{eff}, \mathrm{n}}}{l_{y}} \frac{\partial}{\partial Y} \Phi_{1, n}\right]=a_{n} F j_{n}$

$\left.\frac{\partial \Phi_{1, n}}{\partial X}\right|_{X=0}=0$

$\left.l_{y} \int_{0}^{1}\left(\frac{1}{l_{p}} \frac{\partial \Phi_{1, n}}{\partial X}\right) d Y\right|_{X=1}=-\frac{I}{\sigma_{e f f, n}}$

$\left.\frac{\partial \Phi_{1, n}}{\partial Y}\right|_{Y=0}=0$

$\left.\frac{\partial \Phi_{1, n}}{\partial Y}\right|_{Y=1}=0$

$\frac{\partial c_{n}^{s}}{\partial t}=\frac{1}{r^{2}} \frac{\partial}{\partial r}\left[r^{2} D_{n}^{s} \frac{\partial c_{n}^{s}}{\partial r}\right]$

$\left.\frac{\partial c_{n}^{s}}{\partial r}\right|_{r=0}=0$

$-\left.D_{n}^{s} \frac{\partial c_{n}^{s}}{\partial r}\right|_{r=R_{s}}=j_{n}$

$\rho_{n} C_{p, n} \frac{d T_{n}}{d t}=\frac{1}{l_{n}} \frac{\partial}{\partial X}\left[\frac{\lambda_{n}}{l_{n}} \frac{\partial T_{n}}{\partial X}\right]+\frac{1}{l_{y}} \frac{\partial}{\partial Y}\left[\frac{\lambda_{n}}{l_{y}} \frac{\partial T_{n}}{\partial Y}\right]$

$-\left.\frac{\lambda_{s}}{l_{s}} \frac{\partial T_{s}}{\partial X}\right|_{X=1}=-\left.\frac{\lambda_{n}}{l_{n}} \frac{\partial T_{n}}{\partial X}\right|_{X=0}$

$+Q_{\mathrm{rxn}, \mathrm{n}}+Q_{\mathrm{rev}, \mathrm{n}}+Q_{\mathrm{ohm}, \mathrm{n}}$

$$
\begin{aligned}
& \left.\frac{\partial T_{n}}{\partial X}\right|_{X=1}=\left.0 T_{n}\right|_{Y=0}=293 K \\
& \left.\frac{\partial T_{n}}{\partial Y}\right|_{Y=1}=0
\end{aligned}
$$

simulated discharge curve for a $1 \mathrm{C}$ discharge rate with a cold plate boundary is given in Figure 2, as well as fully insulated conditions, and applied temperature boundary conditions for comparison.

There is a clear difference in the performance between the three simulations due to the temperature profiles. Furthermore, the cold plate model predicts a discharge curve largely between the two extremes, as would be expected. The temperature profiles arising from

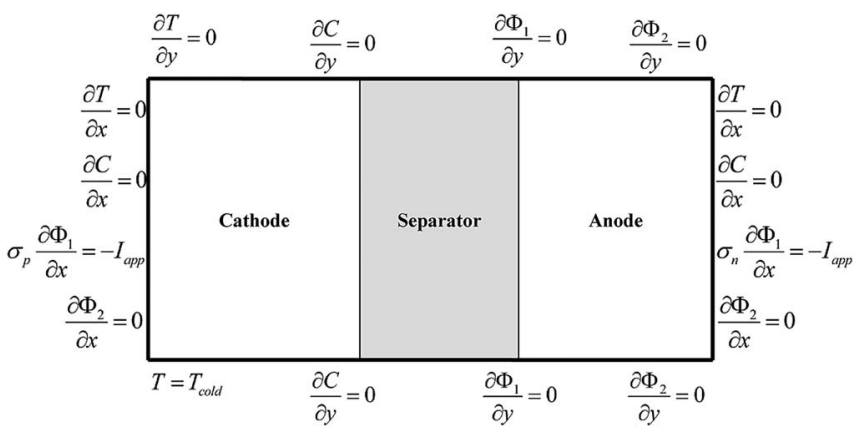

Figure 1. Boundary conditions used as an initial approximation of the $2 \mathrm{D}$ model. the cold plate boundary condition are given in Figure 3 at increasing levels of depth of discharge across the height of the cell. Note that although a true $2 \mathrm{D}$ model is used, the temperature variation across the cell thickness is negligible, so a $1 \mathrm{D}$ plot is shown here. In general, there is a significant temperature gradient near the cold plate, while the temperature is rather flat in roughly half the cell. Additionally, the temperature profile given in Figure 3 can induce a variation in $y$-direction of the other variables, which effects the discharge curves shown in Figure 2.

Figure 4 shows the contour plots of the pore wall flux in the cathode at four points during the discharge. This shows that a variation in the $y$-direction can occur in the other variables. The higher temperatures affect the diffusion of lithium, as well as the rates of reaction, leading to variation in the local pore wall flux. This is important as this can lead to local variation in capacity fade due to increased SEI growth and mechanical stress, which could not be captured in a 1D model.

Two-dimensional thermal lithium-ion cell with $\mathrm{y}$-variation of current density.- The 2D model presented in Figure 1 does not consider the possible variation in the $y$-direction for current density, instead specifying a constant value at the current collector. Under most conditions the variation is likely to be minor, but under certain circumstances, specifically those which result in a thermal gradient across 


$$
\begin{aligned}
& \text { Table III. Additional Equations. } \\
& Q_{\mathrm{rxn}, i}=F a_{i} j_{i}\left(\Phi_{1, i}-\Phi_{2, i}-U_{i}\right), i=p, n \\
& Q_{\mathrm{rev}, i}=F a_{i} j_{i} T_{i} \frac{\partial U_{i}}{\partial T}, i=p, n \\
& Q_{\mathrm{ohm}, i}=\sigma_{\mathrm{eff}, i}\left(\frac{1}{l_{i}} \frac{\partial \Phi_{1, i}}{\partial X}\right)^{2}+\kappa_{\mathrm{eff}, i}\left(\frac{1}{l_{i}} \frac{\partial \Phi_{2, i}}{\partial X}\right)^{2}+\frac{2 \kappa_{\mathrm{eff}, i} R T_{i}}{F}\left(1-t_{+}^{0}\right) \frac{1}{l_{i}^{2}} \frac{1}{c_{i}} \frac{\partial c_{i}}{\partial X} \frac{\partial \Phi_{2, i}}{\partial X}, i=p, n \\
& Q_{\mathrm{ohm}, \mathrm{s}}=\kappa_{\mathrm{eff}, s}\left(\frac{1}{l_{s}} \frac{\partial \Phi_{2, s}}{\partial x}\right)^{2}+\frac{2 \kappa_{\mathrm{eff}, s} R T_{s}}{F}\left(1-t_{+}^{0}\right) \frac{1}{c_{s}} \frac{1}{l_{s}^{2}} \frac{\partial c_{s}}{\partial X} \frac{\partial \Phi_{2, i}}{\partial X} \\
& D_{\text {eff }, i}=\varepsilon_{i}^{\text {brugg }_{i}} 1 \times 10^{-4} \times 10^{-4.43-\frac{54}{T_{i}-229-5.0 \times 10^{-3} c_{i}}-0.22 \times 10^{-3} c_{i}}, i=p, s, n \\
& \kappa_{i}=\varepsilon_{i}^{\text {brugg }_{i}} 1.0 \times 10^{-4} \times c_{e, i}\left(\begin{array}{l}
-10.5+0.668 \times 10^{-3} c_{i}+0.494 \times 10^{-6} c_{i}^{2}+0.074 T_{i}- \\
1.78 \times 10^{-5} c_{i} T_{i}-8.86 \times 10^{-10} c_{i}^{2} T_{i}-6.96 \times 10^{-5} T_{i}^{2}+2.80 \times 10^{-8} c_{i} T_{i}^{2}
\end{array}\right)^{2} \\
& U_{i}\left(T_{i}, \theta_{i}\right)=U_{\mathrm{i}, \mathrm{ref}}\left(T_{\mathrm{ref}}, \theta_{i}\right)+\left.\left(T_{i}-T_{\mathrm{ref}}\right)\left[\frac{d U_{i}}{d T}\right]\right|_{T_{\mathrm{ref}}}, i=p, n \\
& \frac{d U_{p}}{d T}=-0.001 \frac{\left[\begin{array}{l}
0.199521039-0.928373822 \theta_{p}+ \\
1.364550689000003 \theta_{p}^{2}-0.6115448939999998 \theta_{p}^{3}
\end{array}\right]}{\left[\begin{array}{l}
\left(1-5.661479886999997 \theta_{p}+11.47636191 \theta_{p}^{2}-\right. \\
\left.9.82431213599998 \theta_{p}^{3}+3.048755063 \theta_{p}^{4}\right)
\end{array}\right]} \\
& \frac{d U_{n}}{d T}=\frac{0.001\left[\begin{array}{l}
0.005269056+3.299265709 \theta_{n}-91.79325798 \theta_{n}^{2}+1004.911008 \theta_{n}^{3}-5812.278127 \theta_{n}^{4}+ \\
19329.7549 \theta_{n}^{5}-37147.8947 \theta_{n}^{6}-38379.18127 \theta_{n}^{7}-16515.05308 \theta_{n}^{8}
\end{array}\right]}{\left[\begin{array}{l}
1-48.09287227 \theta_{n}+1017.234804 \theta_{n}^{2}-10481.80419 \theta_{n}^{3}+59431.3 \theta_{n}^{4}- \\
195881.6488 \theta_{n}^{5}+374577.3152 \theta_{n}^{6}-385821.1607 \theta_{n}^{7}+165705.8597 \theta_{n}^{8}
\end{array}\right]} \\
& D_{i, e f f}^{s}=D_{i}^{s} \exp \left(-\frac{E_{a}^{D_{i}^{s}}}{R}\left[\frac{1}{T}-\frac{1}{T_{\text {ref }}}\right]\right), i=p, n \\
& k_{i, e f f}=k_{i} \exp \left(-\frac{E_{a}^{k_{i}}}{R}\left[\frac{1}{T}-\frac{1}{T_{\text {ref }}}\right]\right), i=p, n
\end{aligned}
$$

\begin{tabular}{|c|c|c|c|c|c|}
\hline Symbol & Parameter & $\begin{array}{l}\text { Positive } \\
\text { Electrode }\end{array}$ & Separator & $\begin{array}{l}\text { Negative } \\
\text { Electrode }\end{array}$ & Units \\
\hline$\overline{\sigma_{i}}$ & Solid phase conductivity & 100 & & 100 & $\mathrm{~S} / \mathrm{m}$ \\
\hline$\varepsilon_{f, i}$ & Filler fraction & 0.025 & & 0.0326 & \\
\hline$\varepsilon_{i}$ & Porosity & 0.385 & 0.724 & 0.485 & \\
\hline Brugg & Bruggman Coefficient & & 4 & & \\
\hline$D$ & Electrolyte diffusivity & $7.5 \times 10^{-10}$ & $7.5 \times 10^{-10}$ & $7.5 \times 10^{-10}$ & $\mathrm{~m}^{2} / \mathrm{s}$ \\
\hline$D_{i}^{s}$ & Solid Phase Diffusivity & $1.0 \times 10^{-14}$ & & $3.9 \times 10^{-14}$ & $\mathrm{~m}^{2} / \mathrm{s}$ \\
\hline$k_{i}{ }_{i}$ & Reaction Rate constant & $2.334 \times 10^{-11}$ & & $5.031 \times 10^{-11}$ & $\mathrm{~mol} /\left(\mathrm{s} \mathrm{m}^{2}\right) /\left(\mathrm{mol} / \mathrm{m}^{3}\right)^{1+\alpha \mathrm{a}, \mathrm{i}}$ \\
\hline$c_{i, \max }^{s}$ & Maximum solid phase concentration & 51554 & & 30555 & $\mathrm{~mol} / \mathrm{m}^{3}$ \\
\hline $\begin{array}{c}l, \max \\
c_{i, 0}^{s}\end{array}$ & Initial solid phase concentration & 25751 & & 26128 & $\mathrm{~mol} / \mathrm{m}^{3}$ \\
\hline$c_{0}^{l, 0}$ & Initial electrolyte concentration & & 1000 & & $\mathrm{~mol} / \mathrm{m}^{3}$ \\
\hline$R_{p, i}$ & Particle Radius & $2.0 \times 10^{-6}$ & & $2.0 \times 10^{-6}$ & M \\
\hline$a_{i}, t$ & Particle Surface Area to Volume & 885000 & & 723600 & $\mathrm{~m}^{2} / \mathrm{m}^{3}$ \\
\hline$l_{i}$ & Region thickness & $80 \times 10^{-6}$ & $25 \times 10^{-6}$ & $88 \times 10^{-6}$ & $\mathrm{M}$ \\
\hline$t_{+}$ & Transference number & & 0.364 & & \\
\hline$F$ & Faraday's Constant & & 96487 & & $\mathrm{C} / \mathrm{mol}$ \\
\hline$R$ & Gas Constant & & 8.314 & & $\mathrm{~J} /(\mathrm{mol} \mathrm{K})$ \\
\hline$T_{\text {ref }}$ & Temperature & & 298.15 & & $\mathrm{~K}$ \\
\hline$\rho$ & Density & 2500 & 1100 & 2500 & $\mathrm{~kg} / \mathrm{m}^{3}$ \\
\hline$C_{p}$ & Specific Heat & 700 & 700 & 700 & $\mathrm{~J} /(\mathrm{kg} \mathrm{K})$ \\
\hline$\lambda$ & Thermal Conductivity & 2.1 & 0.16 & 1.7 & $\mathrm{~J} /(\mathrm{m} \mathrm{K})$ \\
\hline$E_{a}^{D_{i}^{s}}$ & Activation Energy for Temperature Dependent Solid Phase Diffusion & 5000 & & 5000 & $\mathrm{~J} / \mathrm{mol}$ \\
\hline$E_{a}^{k_{i}}$ & Activation Energy for Temperature Dependent Reaction Constant & 5000 & & 5000 & $\mathrm{~J} / \mathrm{mol}$ \\
\hline
\end{tabular}

\section{Table IV. Parameter values.}




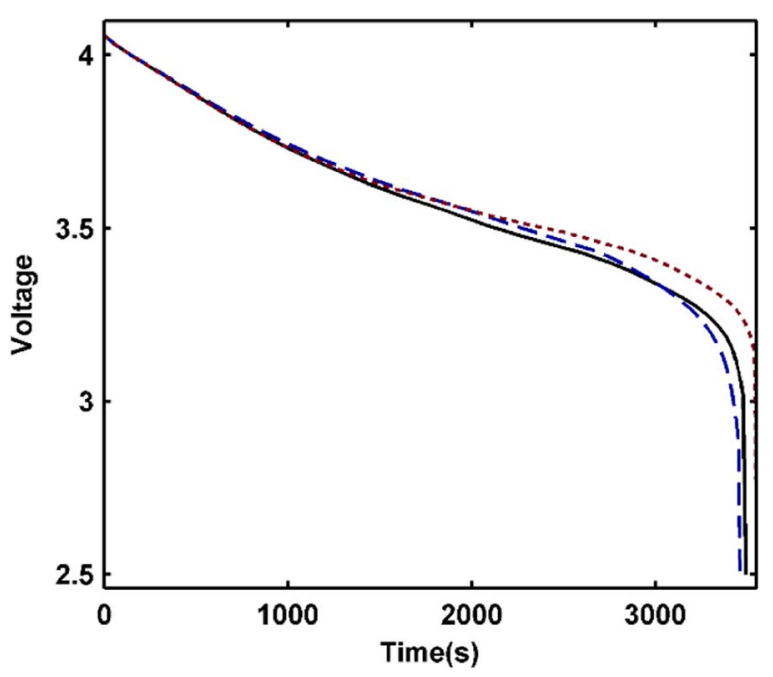

Figure 2. Discharge curve using the P3D model with cold plate boundary conditions (solid line) compared to fully insulated 2D model (long dash line) and a 2D model with fixed temperature boundary conditions on all sides (short dash line).

the height of the battery, the current density may not be constant, which can occur in large format cells. As an initial approach at relaxing the constant current density assumption, a constant current charge (or discharge) is simulated by requiring that the integral of the current density across the current collector is a constant, as demonstrated

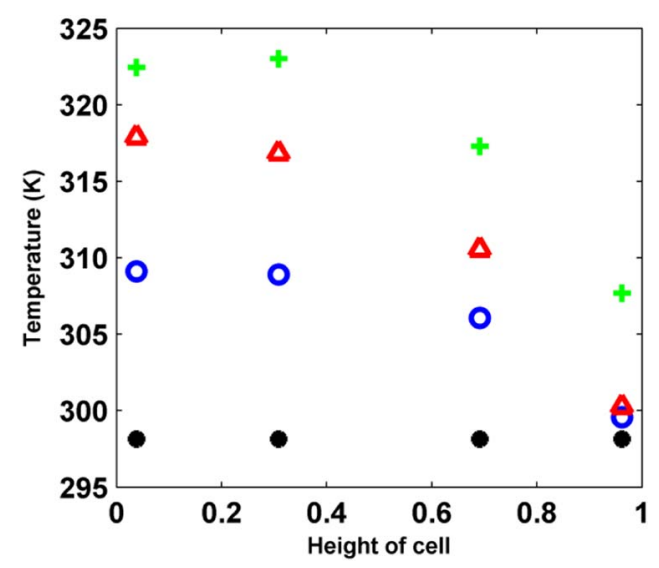

Figure 3. Temperature across cell height at $0 \%$ DOD (black ' $*$ '), 33\% DOD (blue 'o'), 67\% DOD (red ' $\Delta$ '), and 100\% DOD (green '+').

in Figure 5.

$$
\int_{0}^{l_{y}} \sigma \frac{\partial \Phi_{1}}{\partial x} d y=-I_{a p p}
$$

Thus, although the current density may vary at different points in $y$, the total current flowing through the cell is constant. However, the boundary condition given in Equation 2 is not adequate to fully characterize the system, as an infinite number of profiles can satisfy the condition. Therefore, the assumption is made that the solid phase potential at the (a)

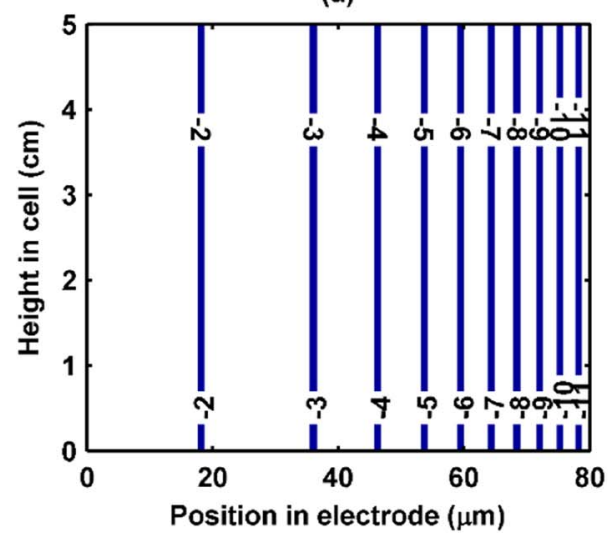

(c)

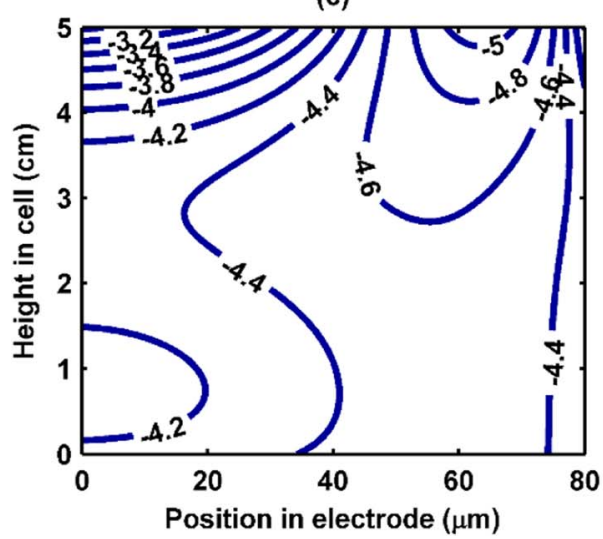

(b)

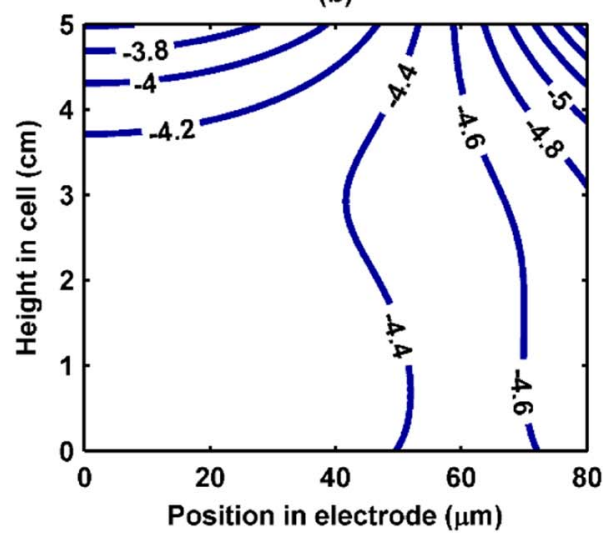

(d)

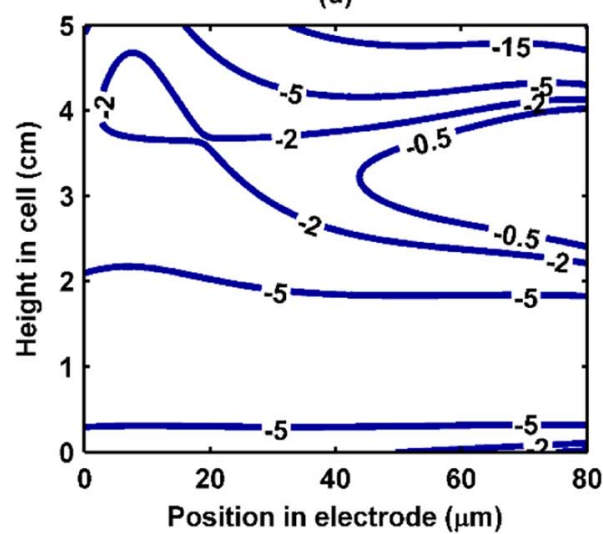

Figure 4. Contour plots of the pore wall flux in the cathode at 0\% DOD (a), 33\% DOD (b), 67\% DOD (c), and 100\% DOD (d). Note that the contour lines are in units of $\frac{\mu \mathrm{mol}}{\mathrm{m}^{2} \mathrm{~s}} \cdot x=0$ corresponds to the cathode-current collector interface and $y=5$ corresponds to the fixed temperature boundary condition. 


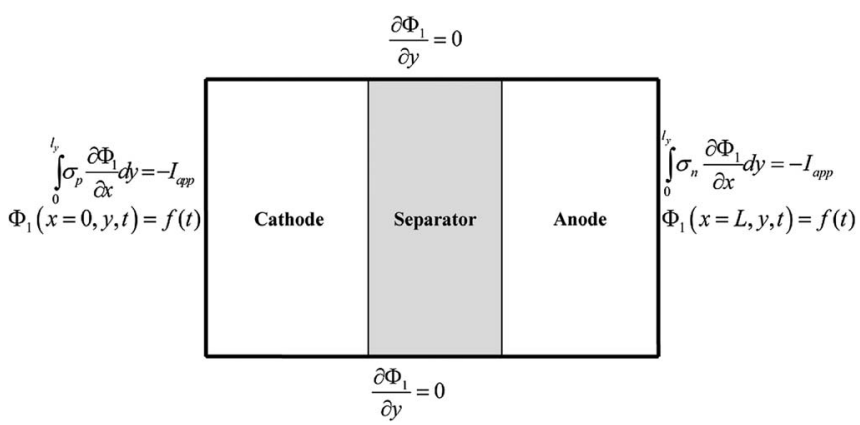

Figure 5. Alternate boundary conditions for the $2 \mathrm{D}$ battery model.

current collector interface does not vary across the height of the cell, although it is allowed to vary with time. Mathematically this can be expressed as:

$$
\Phi_{1}(x=0, y, t)=f(t)
$$

This assumption is considered valid as the high electric conductivities of the current collectors (typically constructed of aluminum and copper) ensure that any variation in voltage potentials is minimal. Thus, the assumption of a constant potential (in $y$ ) is a better assumption to use than a constant current density assumption in most applications.

The boundary conditions given in Equations 12 and 13 have been used to solve for the 2D battery model using reformulation techniques. This approach is robust and allows for implementation of porous electrode models for application in which variation across the height of the cell is expected to play a major role in battery life and performance.

The benchmarking of the reformulated model is done by comparing with the results obtained by solving the model equations using COMSOL Multiphysics. ${ }^{64}$ Both external (voltage-time curve) and internal variables (liquid phase concentration, temperature, etc.) are expected to exactly match for all discharge rates. We define an aspect ratio as the ratio of the length of cell to the height of cell. Figure 6 shows the comparison of the discharge curves for $1 \mathrm{C}, 2 \mathrm{C}$ and $5 \mathrm{C}$ discharge rates for an aspect ratio close to 3:1. It can be seen that the results obtained by the reformulated model are in good agreement with the results predicted by COMSOL Multiphysics using finite element method. To match the internal profiles, liquid phase concentration at $y$ $=\mathrm{H} / 2$ (middle of the cell) vs position ( $\mathrm{x}$ ) and temperature at $\mathrm{x}=0 \mathrm{vs}$ position (y) are also compared. The comparison of concentration and

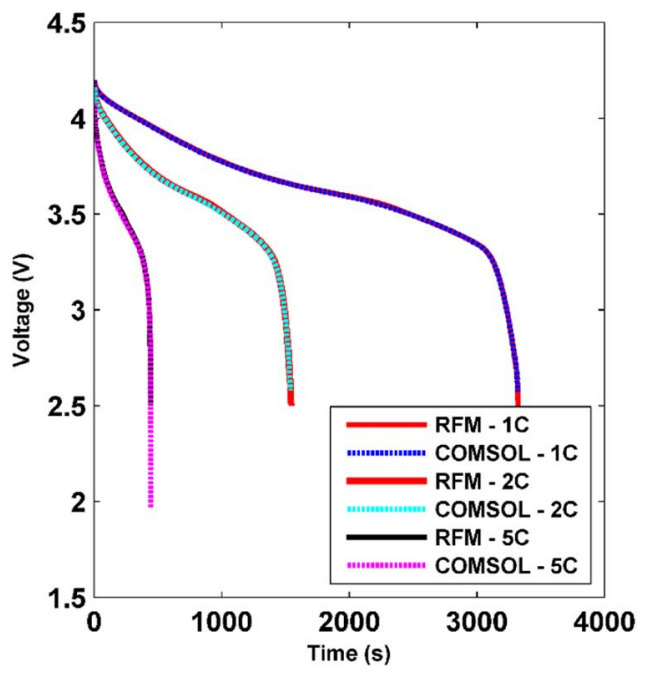

Figure 6. Discharge curves for $1 \mathrm{C}, 2 \mathrm{C}$ and $5 \mathrm{C}$ discharge rates for aspect ratio $3: 1$.

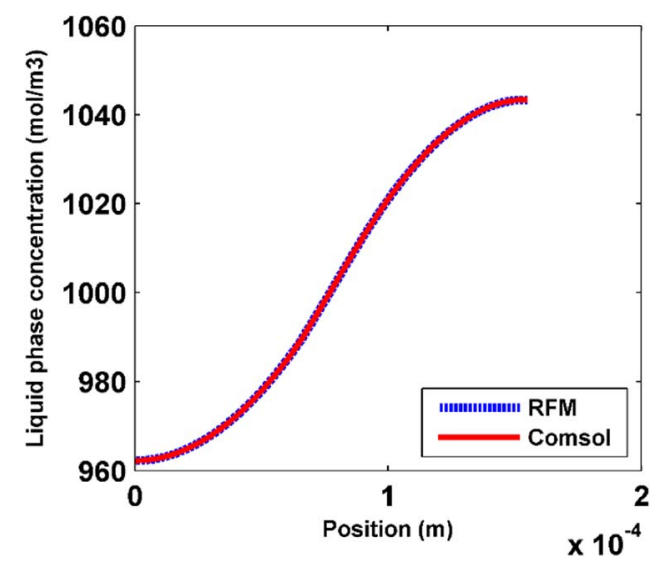

Figure 7. Liquid phase concentration vs position at $y=H / 2$ (mid) for $1 \mathrm{C}$ discharge rate and 3:1 aspect ratio.

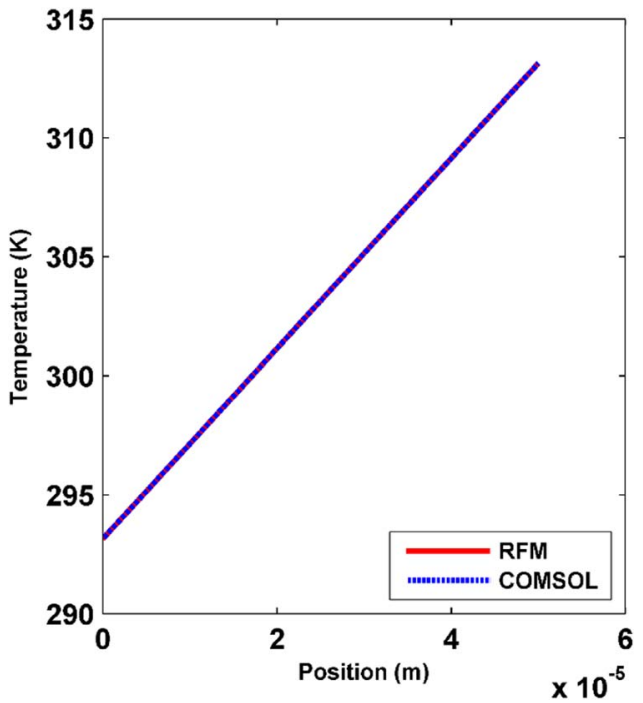

Figure 8. Temperature vs position at $\mathrm{x}=0$ for $1 \mathrm{C}$ discharge rate and 3:1 aspect ratio.

temperature profiles is shown in Figure 7 and Figure 8 respectively for 1C discharge rate and aspect ratio close to $3: 1$.

To validate the model for other aspect ratios, the discharge curves are compared by varying the aspect ratio of the cell. Figure 9 and Figure 10 show the comparison of the discharge curves for an aspect ratio close to 3:10 and 3:25 respectively for $1 \mathrm{C}$ discharge rate. For an

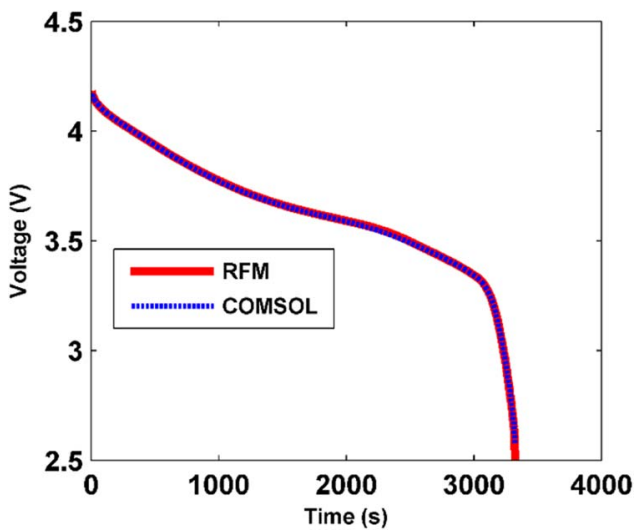

Figure 9. Discharge curve for 1C discharge rate for aspect ratio 3:10. 


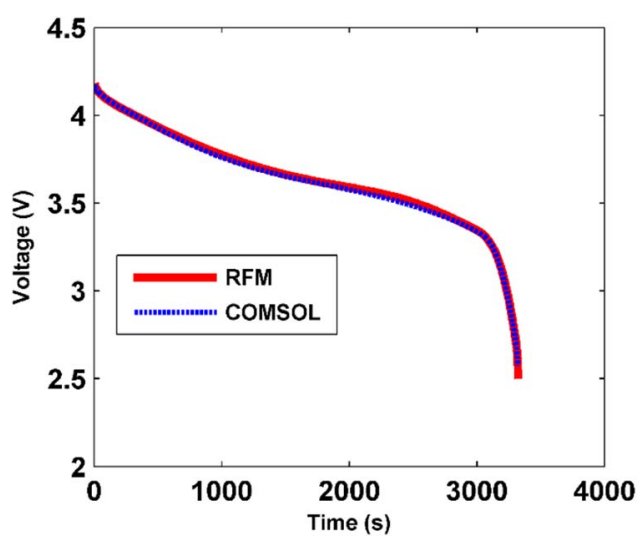

Figure 10. Discharge curve for $1 \mathrm{C}$ discharge rate for aspect ratio 3:25.

aspect ratio greater than $3: 25$, the inbuilt battery module of COMSOL Multiphysics fails to converge with its default solver settings.

Due to the inability of COMSOL to handle large aspect ratios, which are on the order of 1:1000 in a real battery, COMSOL cannot be reliably used to validate the reformulated model in such systems. Therefore, here we compare the RFM results by increasing the number of collocation points to show convergence.

$\mathrm{N}_{\mathrm{C}}$ denotes the number of collocation points used in cathode, $\mathrm{N}_{\mathrm{S}}$ denotes the number of points used in separator, $\mathrm{N}_{\mathrm{A}^{-}}$the number of points used in anode, $\mathrm{N}_{\mathrm{y}}$ - the number of points used in $\mathrm{y}$-direction and $\mathrm{N}_{\mathrm{r}}$ as the number of points used in radial direction. Figure 11 and Figure 12 show the comparison of the discharge curve obtained for $1 \mathrm{C}$ discharge rate for an aspect ratio 1:100 and 1:1000 respectively, for collocation points in the order $\mathrm{N}_{\mathrm{C}}, \mathrm{N}_{\mathrm{S}}, \mathrm{N}_{\mathrm{A}}, \mathrm{N}_{\mathrm{r}}$ and $\mathrm{N}_{\mathrm{y}}$ as 2, 1, 2, 4, 2 respectively (that is, using up to a $2^{\text {nd }}$ order Chebyshev polynomial in the electrodes, $1^{\text {st }}$ order in the separator, and $4^{\text {th }}$ order in the radial and $2^{\text {nd }}$ order in y-direction) and $3,2,3,4,4$. The voltage time curve matches exactly when compared with the curve obtained by increasing the number of collocation points. Table $\mathrm{V}$ shows the CPU times for the reformulated model for various aspect ratios for different number of collocation points, when IDA $^{66}$ is used as the solver in Maple. It can be concluded that the reformulated model gives accurate and faster results for all aspect ratios and an increase in the number of collocation points in any direction has no effect on the accuracy of the results.

The boundary conditions shown in Figure 5 can result in large temperature increases within a sufficiently large lithium-ion cell, even when only a $1 \mathrm{C}$ discharge is applied. Note, importantly, that the current collectors and battery casing, etc. are neglected in this model. Including those would likely reduce the magnitude of the temperature

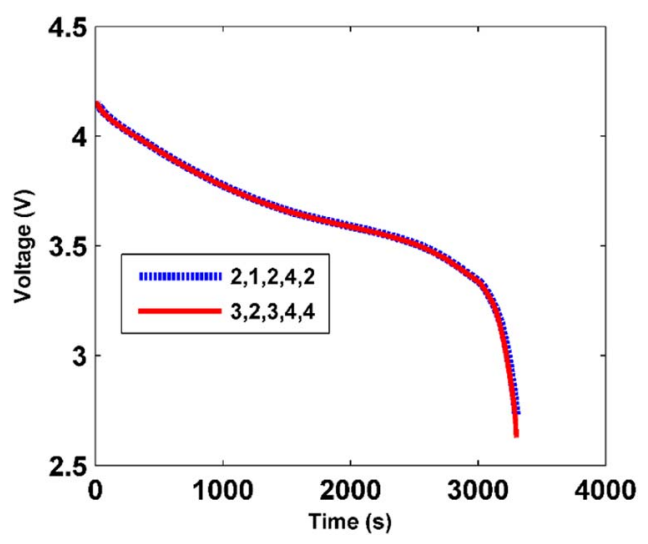

Figure 11. Discharge curve for $1 \mathrm{C}$ discharge rate for aspect ratio 1:100 for different number of collocation points.

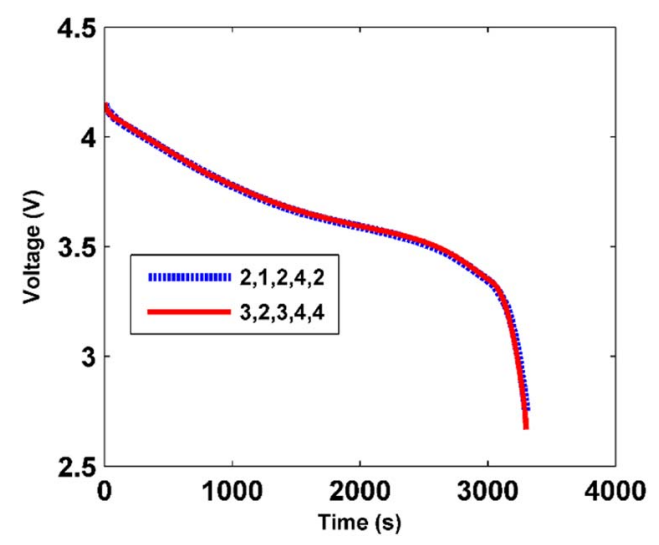

Figure 12. Discharge curve for $1 \mathrm{C}$ discharge rate for aspect ratio 1:1000 for different number of collocation points.

increase by increasing the thermal mass of the system. Also, the current collector, being thermally conductive, would facilitate improved heat transfer across the height of the cell. The temperature increase is shown in Figure 13, as is the current density at both electrode-current collector interfaces, for a cell with a height of $5 \mathrm{~cm}$. The temperature variation across the thickness of the electrode was negligible, as has been observed in the 1D model. Here, the temperature profile in $y$ has a small but noticeable effect on current density for most of the discharge time. Specifically, areas of the cell which are at a higher temperature have a slightly higher current density. A higher temperature facilitates improved diffusion by reducing resistance, and directly increases the rate of reaction. However, at the end of discharge, the current density is provided principally near the applied temperature boundary, and at a much higher rate. This shows that even though the cell as a whole undergoes only a $1 \mathrm{C}$ discharge, the local current density can reach well over $2 \mathrm{C}$ in certain regions of the cell, which was neglected in the first section of this paper. This relatively rapid discharge in a small region of the cell can increase the local capacity fade as well as increasing the local heat generation which in turn can cause a more complicated thermal profile to arise and possibly cause hot spots and further damage to the cell. Here, $(4,1,4,3)$ collocation was used for simulation (that is, using up to a $4^{\text {th }}$ order Chebyshev polynomial in the electrodes, $1^{\text {st }}$ order in the separator, and $3^{\text {rd }}$ order in the $y$-direction).

The changing reaction zone is shown explicitly in Figure 14 which shows the local pore wall flux as contour plots. At the beginning of discharge there is no appreciable variation in the $y$-direction, only across the thickness of the electrode as expected from the 1D model. However, as a greater temperature gradient is established, the variation of the pore wall flux across the height of the cell is more significant than that across the thickness, as shown in Figure 14b and 14c. By the end of discharge, only a small fraction of the electrode volume (near the cold plate and separator) provides the entire current demand. Note that the variation of the pore wall flux in the $y$-direction is more significant in Figure 14 than the case in which the current density variation was ignored and shown in Figure 4.

Table V. CPU time for various aspect ratios for different number of collocation points for IDA solver in Maple.

\begin{tabular}{ccc} 
Aspect Ratio & Number of Collocation Points & CPU Time (Seconds) \\
\hline \multirow{2}{*}{$1: 10$} & $2,1,2,4,4$ & 2.95 \\
$1: 100$ & $3,2,3,4,4$ & 20.38 \\
& $2,1,2,4,2$ & 2.72 \\
$1: 1000$ & $3,2,3,4,4$ & 18.63 \\
& $2,1,2,4,2$ & 2.75 \\
& $3,2,3,4,4$ & 17.32
\end{tabular}



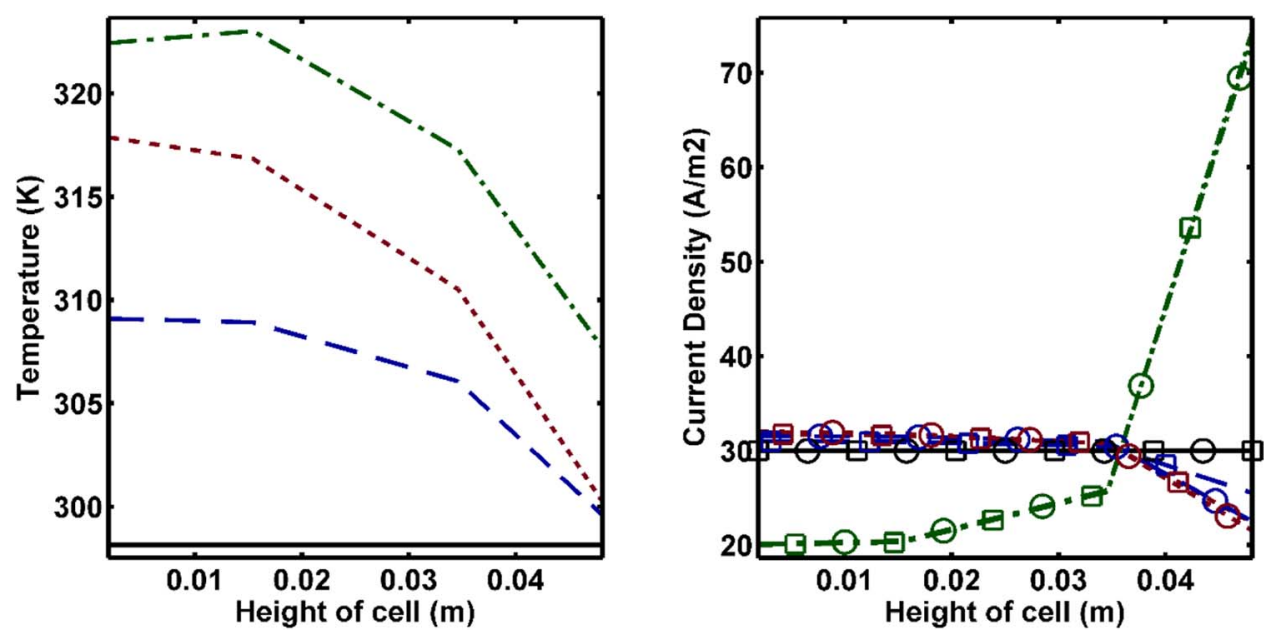

Figure 13. Temperature (left) and current density at the cathode-( $\square$ ) and anode-(o) current collector interfaces (right) for a 1C discharge at $0 \%$ DOD (solid line), $33 \%$ DOD (long dash), 67\% DOD (short dash), and 100\% DOD (dash dot).

The spike in local current density at the end of the discharge can be attributed to the saturation (or depletion, at the anode) of lithium in the warmer areas of the cell. Thus, regions which would not otherwise be favored due to the thermal conditions become favorable sites for reaction for thermodynamic reasons. Figure 15 shows the local degree of lithiation in the cathode. Figure $15 \mathrm{~b}$ and $15 \mathrm{c}$ show that in the midst of discharge, the SOC varies significantly in both the $x$ - and $y$ - directions, and that the variation across the height mirrors the gradient of temperature seen in Figure 13. Ultimately, Figure 15d shows that there is a region of the cell adjacent to the current collector and near the cold plate which is significantly underutilized. In this region, the diffusion and ohmic resistance is at its highest point in the electrode. It is both cooler, directly limiting lithium diffusion, and furthest from the separator, requiring more current to be carried in the electrolyte. (a)

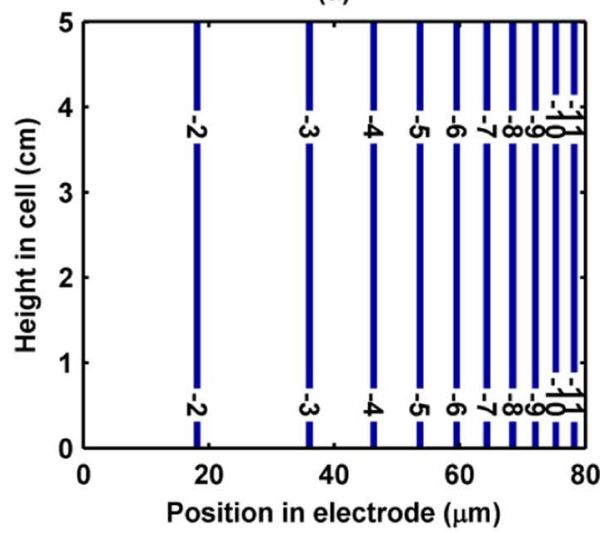

(c)

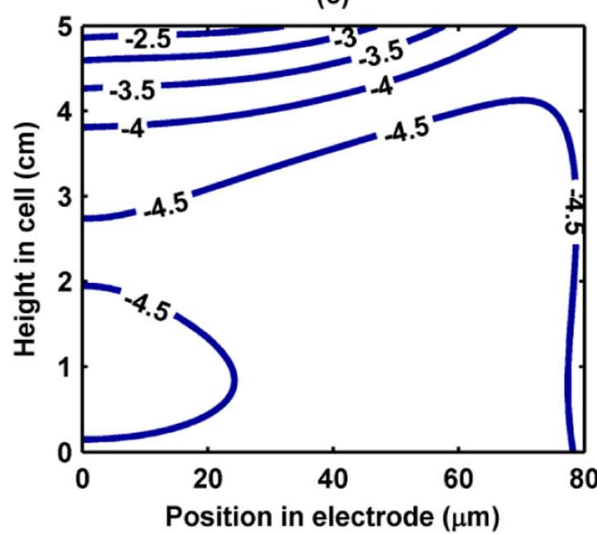

(b)

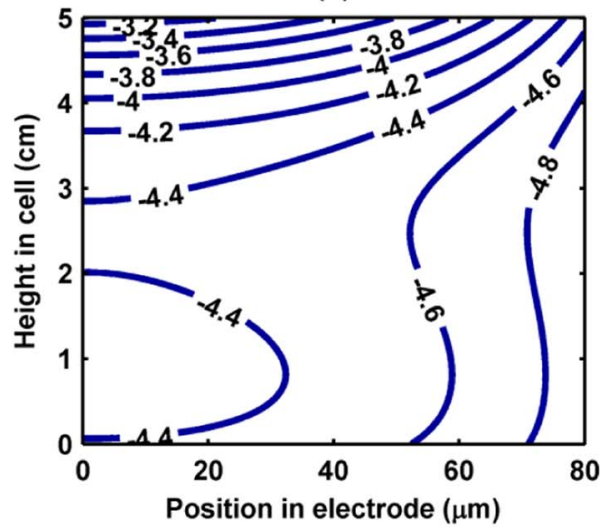

(d)

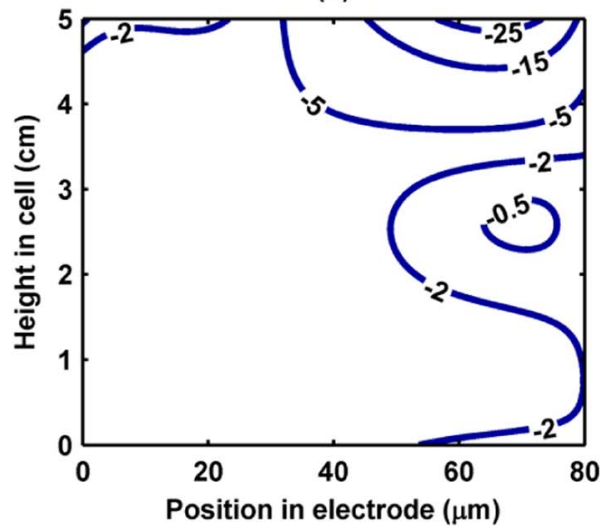

Figure 14. Contour plots of the pore wall flux in the cathode at $0 \%$ DOD (a), 33\% DOD (b), 67\% DOD (c), and 100\% DOD (d). Note that the contour lines are in units of $\frac{\mu \mathrm{mol}}{\mathrm{m}^{2} \mathrm{~s}} \cdot x=0$ corresponds to the cathode-current collector interface and $y=5$ corresponds to the fixed temperature boundary condition. 
(a)

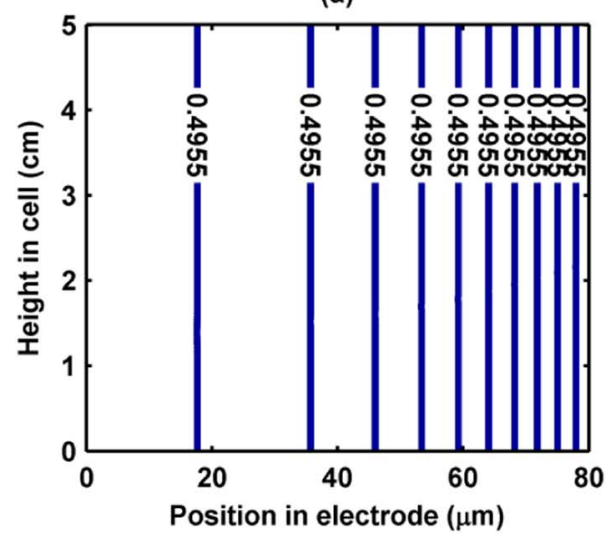

(c)

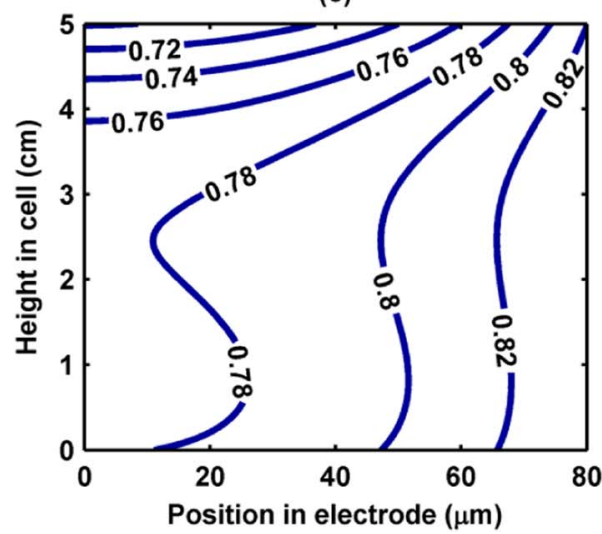

(b)

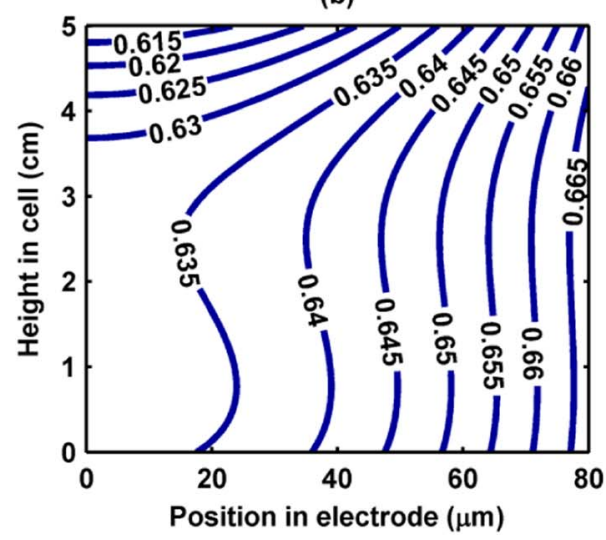

(d)

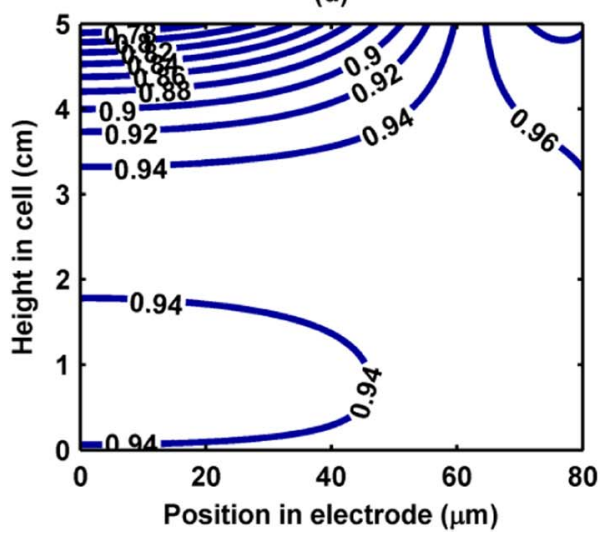

Figure 15. Contour plots of the local degree of lithiation in the cathode at (a) $0 \%$ DOD, (b) $33 \%$ DOD, (c) $67 \%$ DOD, and (d) $100 \%$ DOD. $x=0$ corresponds to the cathode-current collector interface and $y=5$ corresponds to the fixed temperature boundary condition.

\section{Conclusions and Future Directions}

In this paper, a two dimensional battery model was developed and demonstrated using Chebyshev-based orthogonal collocation. This showed that large variations in internal variables can arise, even under relatively mild conditions. In the example considered in this work, the temperature boundary conditions were the only factors which contributed to the asymmetry of the cell performance. However, this asymmetry leads to a very non-uniform utilization of the electrode and local pore wall flux. This causes areas of the electrode to have local current densities to be much higher than expected based on the total current of the cell. This can lead to premature degradation of

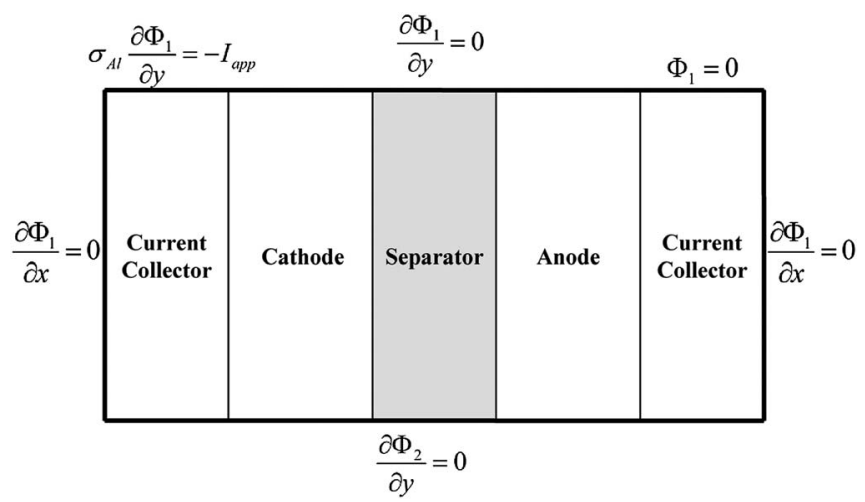

Figure 16. Proposed boundary conditions for the solid phase potential in a 2D model with current collectors included. regions of electrode and can accelerate the capacity fade. By using mathematical models, these conditions can be better predicted and avoided to allow for batteries to be used for longer life.

However, the battery model given in Figure 5 is not fully inclusive as the variation of potential across the battery height is neglected. A natural extension would be the inclusion of the current flow through the current collectors in the model, as shown in Figure 16. Continuity of the solid phase potential and flux would have to be applied at the current collector/electrode interfaces. This would allow for variation of both potential and current density at the current collector-electrode interface. The current out of the current collector could be approximated as having a constant flux at the tab. This formulation would neglect the possible variation in current density across the thickness of the current collector, but that variation is expected to be negligible due to the high conductivity and small thickness of the current collectors.

\section{List of Symbols}

$B_{j, k}(t) \quad$ Collocation coefficients

$c \quad$ Electrolyte concentration

$c^{s} \quad$ Solid Phase Concentration

$D \quad$ Liquid phase Diffusion coefficient

$D_{\text {eff }} \quad$ Effective Diffusion coefficient

$D^{s} \quad$ Solid phase diffusion coefficient

$E_{a} \quad$ Activation Energy

$F \quad$ Faraday's Constant

I Applied Current

$j \quad$ Pore wall flux

$k \quad$ Reaction rate constant

$l \quad$ Length of region

$N \quad$ Number of terms to approximate the solution 


\section{Related to Solid Phase}

\section{References}

1. R. C. Kroeze and P. T. Krein, in Power Electronics Specialists Conference, 2008. PESC 2008. IEEE, p. 1336 (2008).

2. H. Rahimi-Eichi and C. Mo-Yuen, in IECON 2012 - 38th Annual Conference on IEEE Industrial Electronics Society, p. 4012 (2012).

3. M. Doyle, T. F. Fuller, and J. Newman, J. Electrochem. Soc., 140, 1526 (1993).

4. K. Kumaresan, G. Sikha, and R. E. White, J. Electrochem. Soc., 155, A164 (2008).

5. P. W. C. Northrop, P. A. Ramachandran, W. E. Schiesser, and V. R. Subramanian, Chem. Eng. Sci., 90, 32 (2013).

6. G. G. Botte, V. R. Subramanian, and R. E. White, Electrochim. Acta, 45, 2595 (2000)

7. V. Ramadesigan, P. W. C. Northrop, S. De, S. Santhanagopalan, R. D. Braatz, and V. R. Subramanian, J. Electrochem. Soc., 159, R31 (2012).

8. M. B. Pinson and M. Z. Bazant, J. Electrochem. Soc, 160, A243 (2013).

9. M. Guo, G. Sikha, and R. E. White, J. Electrochem. Soc., 158, A122 (2011).

10. S. Santhanagopalan, Q. Z. Guo, P. Ramadass, and R. E. White, J. Power Sources, 156, 620 (2006).

11. D. Zhang, B. N. Popov, and R. E. White, J. Electrochem. Soc., 147, 831 (2000)

12. J. Newman and W. Tiedemann, Aiche J., 21, 25 (1975).

13. P. Arora, M. Doyle, A. S. Gozdz, R. E. White, and J. Newman, J. Power Sources, 88 , 219 (2000).

14. M. Doyle, J. Newman, A. S. Gozdz, C. N. Schmutz, and J. M. Tarascon, J. Electrochem. Soc., 143, 1890 (1996).

15. T. F. Fuller, M. Doyle, and J. Newman, J. Electrochem. Soc., 141, 982 (1994).

16. T. F. Fuller, M. Doyle, and J. Newman, J. Electrochem. Soc., 141, 1 (1994).

17. P. M. Gomadam, J. W. Weidner, R. A. Dougal, and R. E. White, J. Power Sources, 110, 267 (2002).

18. G. Ning, R. E. White, and B. N. Popov, Electrochim. Acta, 51, 2012 (2006).

19. P. Ramadass, B. Haran, P. M. Gomadam, R. White, and B. N. Popov, J. Electrochem. Soc., 151, A196 (2004)

20. P. Ramadass, B. Haran, R. White, and B. N. Popov, J. Power Sources, 123, 230 (2003).

21. K. E. Thomas and J. Newman, J. Electrochem. Soc., 150, A176 (2003).
22. M. Safari, M. Morcrette, A. Teyssot, and C. Delacourt, J. Electrochem. Soc., 156, A145 (2009).

23. M. C. Tucker, J. A. Reimer, and E. J. Cairns, Electrochem. Solid St., 3, 463 (2000)

24. H. F. Wang, Y. I. Jang, B. Y. Huang, D. R. Sadoway, and Y. T. Chiang, J. Electrochem. Soc., 146, 473 (1999).

25. J. Christensen and J. Newman, J. Solid State Electr., 10, 293 (2006).

26. J. Christensen and J. Newman, J. Electrochem. Soc., 153, A1019 (2006).

27. X. C. Zhang, W. Shyy, and A. M. Sastry, J. Electrochem. Soc., 154, A910 (2007).

28. S. Renganathan, G. Sikha, S. Santhanagopalan, and R. E. White, J. Electrochem. Soc., 157, A155 (2010).

29. R. E. Garcia, Y. M. Chiang, W. C. Carter, P. Limthongkul, and C. M. Bishop, J. Electrochem. Soc., 152, A255 (2005).

30. T. M. Bandhauer, S. Garimella, and T. F. Fuller, J. Electrochem. Soc., 158, R1 (2011).

31. T. M. Bandhauer, S. Garimella, and T. F. Fuller, J. Power Sources, 247, 618 (2014).

32. D. Bernardi, E. Pawlikowski, and J. Newman, J. Electrochem. Soc., 132, 5 (1985).

33. C. R. Pals and J. Newman, J. Electrochem. Soc., 142, 3274 (1995).

34. L. Rao and J. Newman, J. Electrochem. Soc., 144, 2697 (1997).

35. L. Song and J. W. Evans, J. Electrochem. Soc., 147, 2086 (2000).

36. W. B. Gu and C. Y. Wang, J. Electrochem. Soc., 147, 2910 (2000).

37. W. E. Schiesser, The numerical method of lines: integration of partial differential equations, Academic Press (1991).

38. V. R. Subramanian, V. Boovaragavan, V. Ramadesigan, and M. Arabandi, J. Electrochem. Soc., 156, A260 (2009).

39. V. R. Subramanian, V. D. Diwakar, and D. Tapriyal, J. Electrochem. Soc., 152, A2002 (2005).

40. P. W. C. Northrop, V. Ramadesigan, S. De, and V. R. Subramanian, J. Electrochem. Soc., 158, A1461 (2011).

41. K. E. Brenan, S. L. Campbell, S. L. V. Campbell, and L. R. Petzold, Numerical solution of initial-value problems in differential-algebraic equations, Society for Industrial and Applied Mathematics (1996).

42. R. N. Methekar, V. Ramadesigan, J. C. Pirkle, and V. R. Subramanian, Comput. Chem. Eng., 35, 2227 (2011).

43. V. Ramadesigan, V. Boovaragavan, J. C. Pirkle, and V. R. Subramanian, J. Electrochem. Soc, 157, A854 (2010).

44. K. Smith and C. Y. Wang, J. Power Sources, 161, 628 (2006).

45. C. Y. Wang, W. B. Gu, and B. Y. Liaw, J. Electrochem. Soc., 145, 3407 (1998).

46. Y. Zeng, P. Albertus, R. Klein, N. Chaturvedi, A. Kojic, M. Z. Bazant, and J. Christensen, J. Electrochem. Soc., 160, A1565 (2013).

47. L. Cai and R. E. White, J. Electrochem. Soc., 156, A154 (2009).

48. S. I. Lee, Y. S. Kim, and H. S. Chun, Electrochim. Acta, 47, 1055 (2002).

49. V. Ramadesigan, K. J. Chen, N. A. Burns, V. Boovaragavan, R. D. Braatz, and V. R. Subramanian, J. Electrochem. Soc., 158, A1048 (2011).

50. S. De, P. W. C. Northrop, V. Ramadesigan, and V. R. Subramanian, J. Power Sources, 227, 161 (2013)

51. B. Suthar, V. Ramadesigan, P. W. C. Northrop, B. Gopaluni, S. Santhanagopalan, R. D. Braatz, and V. R. Subramanian, in American Control Conference (ACC), 2013 , p. 5350 (2013).

52. Y. F. Chen and J. W. Evans, J. Electrochem. Soc., 141, 2947 (1994)

53. C. R. Pals and J. Newman, J. Electrochem. Soc., 142, 3282 (1995).

54. U. S. Kim, C. B. Shin, and C. S. Kim, J. Power Sources, 189, 841 (2009).

55. U. S. Kim, J. Yi, C. B. Shin, T. Han, and S. Park, J. Electrochem. Soc., 160, A990 (2013).

56. Y. Inui, Y. Kobayashi, Y. Watanabe, Y. Watase, and Y. Kitamura, Energ. Convers. Manage., 48, 2103 (2007).

57. G. H. Kim, K. Smith, K. J. Lee, S. Santhanagopalan, and A. Pesaran, J. Electrochem. Soc., 158, A955 (2011).

58. R. E. Gerver and J. P. Meyers, J. Electrochem. Soc., 158, A835 (2011)

59. D. A. H. MeCleary, J. P. Meyers, and B. Kim, J. Electrochem. Soc., 160, A1931 (2013).

60. J. Christensen, D. Cook, and P. Albertus, J. Electrochem. Soc., 160, A2258 (2013).

61. Fluent, [http://www.ansys.com/Products/Simulation+Technology/Fluid+Dynamics/ Fluid+Dynamics+Products/ANSYS+Fluent, last accessed 2013].

62. A. Tourani, P. White, and P. Ivey, J. Power Sources, 255, 360 (2014)

63. M. Xu, Z. Zhang, X. Wang, L. Jia, and L. Yang, J. Power Sources, 256, 233 (2014).

64. COMSOL, [http://www.comsol.com, last accessed 2013].

65. J. Villadsen and M. L. Michelsen, Solution of differential equation models by polynomial approximation, Prentice-Hall (1978).

66. IDA, [http://computation.llnl.gov/casc/sundials/main.html, last accessed 2014].

67. J. Wu, V. Srinivasan, J. Xu, and C. Y. Wang, J. Electrochem. Soc., 149(10) A1342 (2002). 